Portland State University

PDXScholar

$6-2018$

\title{
Impaired Fear Recall in a Mouse Model of Traumatic Brain Injury and Post-Traumatic Stress Disorder
}

Natasha Avalon Gardner

Portland State University

Follow this and additional works at: https://pdxscholar.library.pdx.edu/honorstheses

Let us know how access to this document benefits you.

\section{Recommended Citation}

Avalon Gardner, Natasha, "Impaired Fear Recall in a Mouse Model of Traumatic Brain Injury and PostTraumatic Stress Disorder" (2018). University Honors Theses. Paper 598.

https://doi.org/10.15760/honors.608

This Thesis is brought to you for free and open access. It has been accepted for inclusion in University Honors Theses by an authorized administrator of PDXScholar. Please contact us if we can make this document more accessible: pdxscholar@pdx.edu. 
Impaired Fear Recall in a Mouse Model of Traumatic Brain Injury and Post-Traumatic Stress Disorder

by

Natasha Avalon Gardner

An honors thesis submitted in fulfillment of the requirements for the degree of

Bachelor of Science

in

University Honors

$\&$

Psychology

Thesis Research Advisor

Miranda M. Lim MD, PhD

$\&$

Mentor

Carolyn E. Jones PhD

Oregon Health \& Science University and VA Portland Health Care System

Portland State University

2018 


\section{Acknowledgements}

I would like to acknowledge Peyton, Carolyn, Mara, and Miranda for their crucial roles in this study. All played an integral role in the study design. A special thank you to Peyton for performing all the surgeries and to Mara for helping me handle the largest set of data I have ever had to throughout my college career. I am also thankful for the hours of behavior tests that Carolyn and Peyton performed for the sake of this study. I am particularly grateful to my mentors and peers who have shown me endless patience and unwavering willingness to share their wisdom and guidance. Thank you very much.

I also must acknowledge the support, faith, love, and patience my husband and children have shown me throughout this project and my education. Without them I could not make it through all the challenges, they keep me determined. Thank you, family. I must also acknowledge this study's funding, thank you for funding science and a student's professional development! (Supported by: VA BLR\&D Career Development Award (\#IK2 BX002712), VA RR\&D SPiRE Award, Oregon Medical Research Foundation, and Portland VA Research Foundation to MML, and NIH T32AA7468-29 to CEJ). 


\title{
Objective
}

To examine the effect of Traumatic Brain Injury (TBI) and Post-Traumatic Stress Disorder (PTSD) on fear learning, generalization, and extinction.

\begin{abstract}
Traumatic Brain Injury (TBI) and Post-Traumatic Stress Disorder (PTSD) have been studied extensively. However, there is little research examining the interaction between the two. An understanding of this interaction is considered important because it is a common comorbid diagnosis. In this study, we used a mouse model to look at how TBI+PTSD interact to influence contextual fear learning, generalization, and extinction. We employed Controlled Cortical Impact (CCI) and Single Prolonged Stress (SPS) as models of TBI and PTSD, respectively. Fear conditioning and PTSD involve overlapping neural pathways including the amygdala, hippocampus, and medial prefrontal cortex which makes contextual fear conditioning an ideal method for investigating fear learning and behavior in a controlled setting. Four groups (Control, TBI, PTSD, and TBI+PTSD) were analyzed for differences in fear expression during conditioning, context generalization, and fear extinction tests. Although all groups acquired fear equally during fear conditioning, the PTSD group showed increased fear expression during the test for generalization, suggesting a decreased ability to discriminate between aversive and neutral contextual stimuli. Results from extinction tests performed suggest significantly impaired recall of conditioned fear among the TBI+PTSD group in comparison to controls. During fear extinction tests all groups were able to significantly extinguish fear. Ongoing research will further characterize the behavioral phenotype of the combined TBI+PTSD mouse model.
\end{abstract}




\section{Keywords}

Post-Traumatic Stress Disorder, PTSD, Traumatic Brain Injury, TBI, fear conditioning, fear memory, mouse model

\section{Introduction}

In the health field there is interest in developing an understanding of the interaction between Traumatic Brain Injury (TBI) and Post-Traumatic Stress Disorder (PTSD) because of the high rates of co-occurrence (Reger et al., 2012; Meyer, Davies, Barr, Manzerra, \& Forster, 2012). Situations that give rise to a TBI are typically traumatic in nature (examples include motor vehicle crashes or war combat), making the connection between TBI and PTSD clear. For example, one study found that among combat veterans who have been diagnosed with mild TBI, 44\% have also been diagnosed with PTSD (Meyer et al., 2012). Even though TBI and PTSD are commonly diagnosed together in the same individual empirical research is lacking to inform an understanding of this unique behavioral phenotype as well as clinical treatment (McAllister, 2009).

TBI results from an external force that causes damage to the brain (Katz, Cohen, \& Alexander, 2015). The damage may include lacerations and or contusions, fractured skull, internal hemorrhaging, cerebral edema, and or severed never fibers (Bryant, 2011). Damage varies based on the severity of the injury which is rated as mild, moderate, or severe. While several rating scales currently exist, some have proposed that mild TBI is characterized by a loss of consciousness no longer than 30 minutes, moderate TBI is defined as a loss of consciousness 30 minutes to 24 hours, and severe TBI is diagnosed when there is a loss of consciousness lasting longer than 24 hours (Bryant, 2011). Symptoms vary based on TBI severity (Bryant, 2011; Yehuda, 2002). However, there are common symptoms associated with all TBIs which include: 
generalized anxiety, aggressive behavior or irritability, reduced motivation and fatigue, problems with sleep, impaired cognition, increased risk for depression and chronic pain (Katz, Cohen, \& Alexander, 2015; Yehuda 2002). TBI has been identified as a major cause of disability and death that costs the U.S. tens of billions of dollars annually (Katz, Cohen, \& Alexander, 2015; Reger, Puolos, Buen, Giza, Hovda, \& Fanselow, 2011). According to the Centers for Disease Control there are 5.3 million individuals in the U.S. who have been diagnosed with a disabling TBI (Bryant, 2011).

PTSD is classified as an anxiety disorder that may result from exposure to a traumatic event such as combat, rape, or interpersonal violence (Mahan \& Ressler, 2012). Common symptoms associated with PTSD include: re-experiencing the traumatic event through intrusive memories or nightmares, hyperarousal which is the physiological state of heightened vigilance for danger, active avoidance of stimuli associated with the traumatic event, and emotional numbing (Bryant, 2011; Mahan \& Ressler, 2012; Yehuda, 2002). Prevalence rates on PTSD by traumatic event and male or female gender have shown that $65 \%$ of male and $45.9 \%$ female rape survivors, $38.8 \%$ of male combat veterans, and $21.3 \%$ of female victims of physical assault are diagnosed with PTSD (Yehuda, 2002; Richardson, Frueh, \& Acierno, 2010). According to the National Center for PTSD, for every 100 people 7-8 can be expected to develop PTSD at some point in their lifetime and as of 2017, 8 million people live with PTSD. Individuals with PTSD account for the highest rates of healthcare service use, especially because PTSD is commonly misdiagnosed (National Center for PTSD, 2017).

Research has established that both TBI and PTSD are associated with low quality of life, high comorbidity with both psychological and medical disorders, and significant impairments in occupational and social abilities resulting in significant costs on families and communities 
(Yehuda, 2002; Katz, Cohen, \& Alexander, 2015). Furthermore, research suggests that mild TBI is associated with an increased risk for developing psychological disorders such as PTSD by more than two-fold (Bryant, 2011). Evidence suggests that TBI may affect neurological mechanisms crucial for inhibiting learned fear responses from traumatic events (Bryant, 2011; Meyer et al., 2012).

In this study, we examined fear learning, generalization, and extinction specifically, because these are important mechanisms for survival and a good quality of life. Furthermore, we are looking at fear learning because it involves neural circuitry known to be implicated in TBI and PTSD (McAllister, 2009; Mahan \& Ressler, 2012; Meyer et al., 2012; Maren, 2001; Palmer et al., 2016).

Fear learning is a crucial survival mechanism that allows an organism to make associations between adverse events and environmental contexts and cues. This enables the organism to choose the appropriate behavioral response to a given stimuli (Maren, 2001; Maren, Phan \& Liberzon, 2013). Generalization refers to learned associations between traumatic events and contexts being triggered whenever a similar context is encountered because it acts as a reminder of the event. This results in a decreased ability to discriminate between safe and dangerous environments therefore, leading to inappropriate behavioral responses (Mahan \& Ressler, 2012; Maren, Phan \& Liberzon, 2013). Extinction is the process of inhibiting a fear response after learning that the context and or cue is safe through repeated exposure without trauma occurring (Maren, 2001; Maren, Phan \& Liberzon, 2013). Exposure therapy, a common treatment for PTSD, acts the same as fear extinction tests.

Fear conditioning, TBI, and PTSD involve overlapping neural pathways including the amygdala, hippocampus, and prefrontal cortex (PFC) which makes it an ideal method for investigating fear 
learning and behavior in a controlled setting (Mahan \& Ressler, 2012; Reger et al., 2012; Meyer et al., 2012). Importantly, this neural circuitry appears to be conserved across species therefore, findings may be relevant to humans and can be used to inform treatment and to further understand this common comorbid diagnosis (Mahan \& Ressler, 2012; Maren, Phan \& Liberzon, 2013; Palmer et al, 2016). Contextual fear conditioning recruits the hippocampus to encode contextual representations of the testing context, and the cues within it to form an association between the two, in order to predict an adverse outcome (e.g., a foot shock) whereby the association is then projected to the amygdala to illicit a behavioral fear response (Maren, 2001). During fear extinction, the PFC is recruited to initiate an excitatory or inhibitory behavioral response from the amygdala based on a top-down analysis of a context that through repeated exposure without trauma should no longer illicit a fear response (Mahan \& Ressler, 2012; Meyer et al, 2012; Maren, 2001).

To look at fear learning and behavior we used a combined mouse model of TBI and PTSD. For an experimental model of TBI we employed Controlled Cortical Impact (CCI) and for PTSD, Single Prolonged Stress (SPS). The combination of SPS with CCI developed for this study is a novel mouse model of TBI+PTSD. A thorough literature review revealed that researchers have examined fear conditioning in SPS but never in a combined SPS and CCI model like the one proposed in this project. However, prior research has examined the relationship between TBI and PTSD using different combinations of TBI and PTSD models, as well as various tests for examining biological and behavioral changes. Sierra-Mercado et al (2013) used a mouse model of CCI to examine the relationship between TBI and PTSD by testing the particular effect of this TBI model on fear learning and extinction. Xing et al (2013) used Fluid Percussion Injury as a TBI mouse model to investigate the neurobiological mechanisms underlying TBI and PTSD 
pathophysiology. The TBI model Fluid Percussion Injury involves delivering a more concussive type mild traumatic brain injury than the contusive type injury delivered in this study (SierraMercado, D. et al 2013). Acosta et al (2013) used a rat model of CCI combined with a PTSD model, which involved repeated exposure to images of a cat and a 31-day period of social instability, to study the histopathological link between TBI and PTSD. Our study adds to the research examining combined TBI and PTSD by producing a novel combined model to continue studying the pathophysiology of this dual diagnosis, as well as, by deepening an understanding of the effect this combined model has on fear learning and behavior.

\section{Methods}

\section{Animals}

All animal research was done in accordance with the Institutional Animal Care and Use Committee of the Portland VA Healthcare System. Subjects consisted of adult male wildtype C57BL/6J mice (Jackson Laboratory). There was a one-week acclimation time period to the facility before testing began. At the start of testing mice were 10 weeks old. A total sample of 48 mice were randomly assigned to one of four groups: Control, TBI, PTSD, and TBI+PTSD.

\section{Surgeries}

All surgeries and procedures were performed by qualified individuals following aseptic procedures. See Figure 1 for an experimental timeline. 
Figure 1: Experimental Timeline

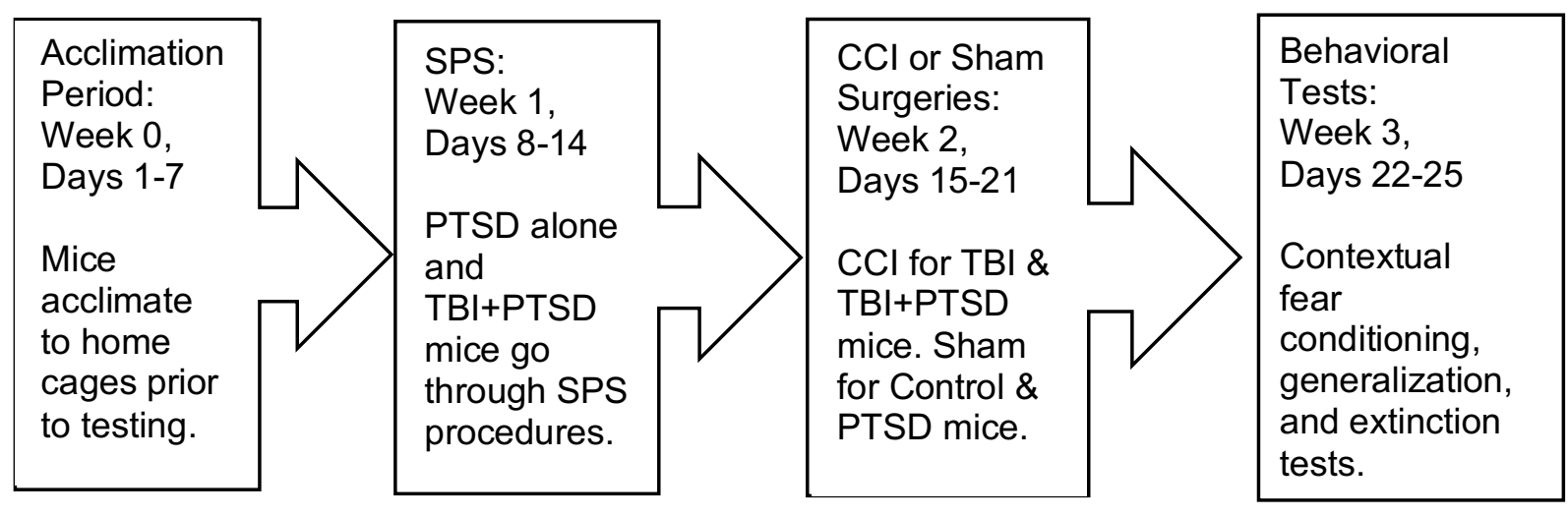

\section{Controlled Cortical Impact (CCI) as a TBI model}

TBI and TBI+PTSD groups underwent CCI. Previous research has validated CCI as an experimental model of TBI (Xiong, Mahmood, \& Chopp, 2013). CCI is a surgical procedure that involves delivering a precise injury to the cortical region. For this study, the depth of the injury was approximately $3.0 \mathrm{~mm}$ delivered at a velocity of $0.5 \mathrm{~m} / \mathrm{s}$ producing a mild to moderate TBI. Mice were weighed prior to surgery and isoflurane was used for anesthesia (3\% induction and $1 \%$ maintenance). Once the appropriate anesthetic plane was reached, the subject was secured in a stereotaxic frame to prevent movement during the surgery. Aseptic technique included shaving the head and then sterilizing with alcohol and iodine. Lidocaine was applied to surgery location then skull landmarks bregma and lambda were exposed via incision. With a cotton applicator, $30 \%$ hydrogen peroxide was used to remove the skull membranes. A $3.0 \mathrm{~mm}$ craniotomy was done between the exposed landmarks, bregma and lambda. An impact was then delivered using Kopf Instruments impactor arm to cortical region at a $3.0 \mathrm{~mm}$ depth. Afterwards, mice received a $1.0 \mathrm{~mL}$ saline injection subcutaneously before being moved to a warming pad in a home cage 
where food, water, and $1.0 \mathrm{~mL}$ of children's acetaminophen in $15 \mathrm{~mL}$ of water were provided. Mice were housed individually after surgery.

\section{Sham Surgery}

Mice in the Control and PTSD groups that did not go through CCI had a sham surgery to control for potential confounds from simply having a surgery. For the sham surgery, mice are anesthetized before being surgically opened revealing the membrane on the skull, which is then removed with $30 \%$ hydrogen peroxide. Afterwards, the scalp was sutured closed.

\section{Single Prolonged Stress (SPS) as a PTSD model}

Previous research has validated SPS as an experimental model of PTSD that involves different stressors that include: a 2-hour tubal restraint, 20-minute forced group swim, ether exposure until loss of consciousness, and finally a seven-day isolation period (Yamamoto et al., 2009; Lim, Song, Yoo, Woo, \& Choe, 2017). For the tube restraint mice were placed in a ventilated 50mL tube and then moved to their home cage for a duration of two hours. Next, mice were placed in groups of four in to plastic tubs filled with room temperature water for 20-minutes. The tubs were big enough to prevent subjects from touching the bottom (8.5"x9.0"x12.0"). Then the mice were dried and moved to glass bell jars with a cotton ball soaked in $1.0 \mathrm{~mL}$ of diethyl ether until they lost consciousness. During the forced group swim and ether exposure mice were closely monitored. Once loss of consciousness occurred mice were moved to home cages where they were socially isolated for a duration of seven days. Subjects that did not go through SPS were group housed during this period. 


\section{Behavioral Tests}

Behavioral tests were recorded with GoPro cameras. Videos were observed by a trained individual who was blind to group. Behavior was scored using Stopwatch+ program (Emory University). Specifically, fear behavior in the form of freezing was scored. Freezing was defined as lack of movement with the exception of the very subtle movement needed for breathing and is a validated method in mouse models to measure fear (Blanchard \& Blanchard, 1969). See Figure 2 for a timeline of behavioral tests performed. 
Figure 2: Timeline of behavioral tests. CS: Conditioned Stimulus (the context), US:

Unconditioned Stimulus (foot-shock).

\section{Day 1: Contextual Fear Conditioning}

Involves repeated pairing of CS (the context) with the US (foot shock) to illicit a behavioral response.

Eight-minute long test.

Three minutes to habituate to context before the first US presentation.

After habituation a one milliamp foot-shock (US) delivered every 60 seconds for a one second duration.

Five total US or foot-shock presentations.

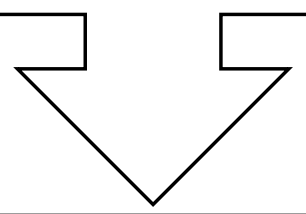

Day 2: Contextual Fear Generalization

Context was altered so as to be experienced as if new. The floor, walls, and scent were all altered.

Subjects were placed in the altered context for a total of three minutes to test generalization of conditioned contextual fear to a neutral context.

No US presentation.

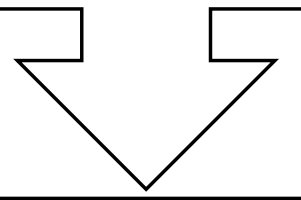

Day 3: Long-Term Recall of Conditioned Fear

Fear behavior (freezing) measured during the first minute mice were re-exposed to the conditioning context to test long-term memory of conditioning.

No US presentation.

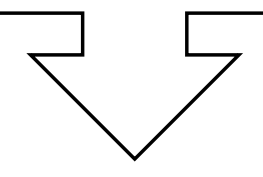

Day 3: Fear Extinction 1

Mice were placed back into the conditioning context.

Test lasted 12 minutes.

No US presentation.

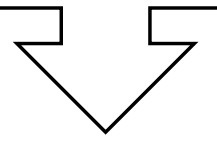

Day 4: Fear Extinction 2

Mice are placed in conditioning context for second fear extinction trial.

Test lasted 12 minutes.

No US presentation. 


\section{Contextual Fear Conditioning (Day 1)}

Fear conditioning entails pairing an aversive stimulus (e.g., a foot-shock) with a neutral stimulus (e.g., a context), whereby later presentation of the context in the absence of the foot-shock elicits a fear response (Maren, 2001). For this study, one-week post-surgery, the subject was placed in the context (conditioned stimulus, CS) for 8 minutes where 5-foot shocks (unconditioned stimulus, US) were delivered at a $1.0 \mathrm{~mA}$ intensity, for 1 second durations, with 60 second intertrial-intervals. The first shock was delivered after a 3-minute habituation period. Subjects were returned to their home cage after test.

The testing chambers that represented the context in this study were from Omnitech Electronics and were $40.8 \mathrm{~cm} \times 14 \mathrm{~cm} \times 18.4 \mathrm{~cm}$ in size. The chamber was well ventilated, and the walls and top was clear acrylic for ease of recording. The flooring consisted of stainless steel rods above a removable waste tray, which was cleaned with water and a paper towel between tests and subjects.

\section{Context Generalization (Day 2)}

Twenty-four hours after fear conditioning the subject was placed in an altered context to test for generalized fear responses and the ability to discriminate between contexts. The walls were covered in black and white checkered paper. The stainless-steel rods were covered with a smooth insert. Additionally, a lemon scent was added by using a scented wipe to clean the waste tray. There was no presentation of the US during this 3-minute test. Subjects were then returned to their home cage.

\section{Fear Recall (Day 3)}

Long-term memory of fear acquisition was examined by comparing the rates of total freezing during the first two-minutes of the first fear extinction test on day three. 


\section{Contextual Fear Extinction (Days 3 \& 4)}

Twenty-four hours after context generalization tests the subject was placed back into the original context, which looked and smelled identical to fear conditioning test day, to look at extinction of acquired fear. The test was performed for a total of 12-minutes without the presentation of US on both days to examine ability. After Day 3 the test subject was returned to their home cage for 24hours and then then the test was performed again on Day 4 (in same manner as Day 3).

\section{Measurement \& Analysis}

Fear behavior was recorded for each test by recording the percent of time (per 1-minute bins) mice spent freezing. Freezing data was compared between groups (i.e. by treatment) and within groups (i.e. by time) using repeated measures of analysis of variance (ANOVAs) statistical methods for tests with multiple time points. For the Context Generalization Test a One-Way ANOVA was conducted to look at treatment effect between subjects. All analyses with significant results were followed up with post-hoc Tukey mean comparison tests. Significance was determined when an alpha value was of 0.05 or less. Original freezing data was collected with Microsoft Excel and then transferred to IBM SPSS to perform analyses. All graphical representations of data were created using GraphPad Prism.

One subject was removed from analyses due to malfunction in foot shock delivery (none were

delivered). Removing a subject from analyses put our PTSD group at a sample size of 11 while all other groups remained at 12 .

\section{Results}

\section{Contextual Fear Conditioning (Day 1)}

There was a significant within subjects effect of US presentation on freezing (repeated measures of ANOVA, within subjects: $F(4,148)=96.947, \mathrm{p}<0.001)$. Initially, subjects showed no fear 
behavior in response to the context prior to US presentation. All subjects acquired a fear response upon the first US presentation and increased freezing across ITIs. An interaction was not observed (repeated measures of ANOVA, group x factor: $F(12,148)=0.573$, p=0.861), as well as, no observed between group effect (repeated measures of ANOVA, between subjects: $F(3,37)=0.884, \mathrm{p}=0.458)$. See Figure 3 .

Figure 3: This graph depicts the mean percent of time different treatment groups spent freezing across ITIs during contextual fear conditioning. There was no difference in the acquisition of fear across the four groups of mice.

Figure 4: Graph shows that PTSD mice expressed significantly more generalized fear from the conditioning context to the neutral context than both control and TBI alone groups.

Figure 3

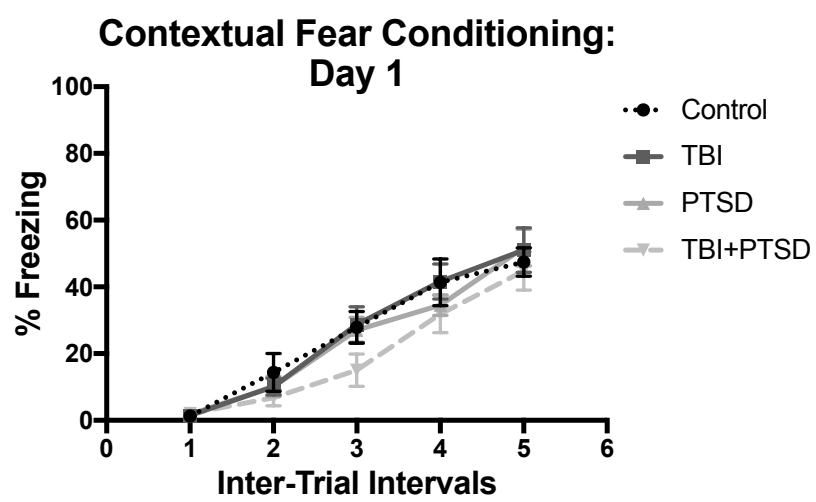

Figure 4

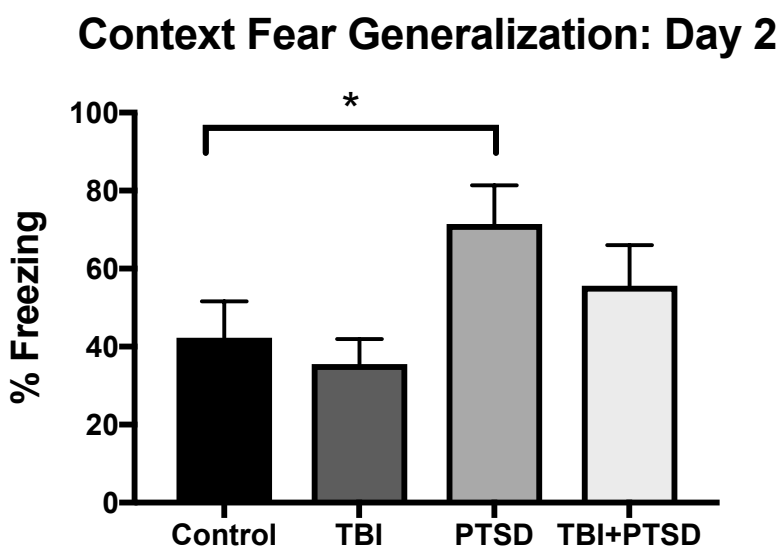

\section{Context Generalization (Day 2)}

There was a significant main effect of treatment group on freezing during the context generalization test (one-way ANOVA, $F(3,43)=2.976$, p=0.042). Groups exhibited different amounts of freezing, or generalizing learned fear, in the altered context. Follow up Tukey mean 
comparisons revealed that the PTSD froze significantly more than the TBI group $(\mathrm{p}=0.04)$. See

Figure 4 .

\section{Fear Recall (Day 3)}

Freezing was analyzed from the first two minutes of being placed back in the conditioning chamber during the extinction tests to examine memory of the US and CS association. There was a significant main effect of fear recall within subjects (repeated measures of ANOVA, $\mathrm{F}(1,43)=14.360, \mathrm{p}<0.001$ ), as well as, between subjects (repeated measures of ANOVA, $\mathrm{F}(3,43)=2.965, \mathrm{p}=0.042)$. Post-hoc Tukey analysis revealed that the effect was driven by the combined TBI+PTSD group, p=0.030. See Figure 5.

Figure 5: Bar graph that shows TBI+PTSD mice exhibited impaired fear recall of previously learned US \& CS association.

Figure 5

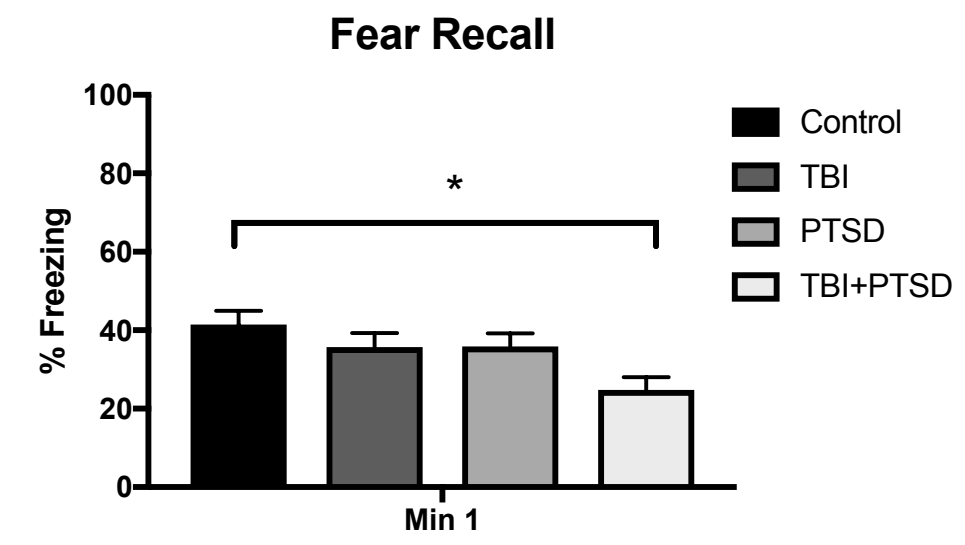




\section{Fear Extinction (Day 3)}

There was a significant main effect of fear extinction within subjects over time (repeated measures ANOVA, $\mathrm{F}(11,473)=41.77, \mathrm{p}<0.001)$ and no effect of group on freezing during extinction was observed (repeated measures ANOVA, between subjects, $F(3,43)=1.73$, $p=0.171$ ). The analysis revealed a group $\mathrm{x}$ time interaction where treatment group influenced how mice responded to the extinction over time (repeated measures ANOVA, $F(33,473)=2.201, p<0.001$ ), driven by the initial low levels of freezing in combined TBI+PTSD. See Figure 6.

Figure 6: Graphs the extinction of learned fear by all treatment groups across the 11-minute test.

Figure 6

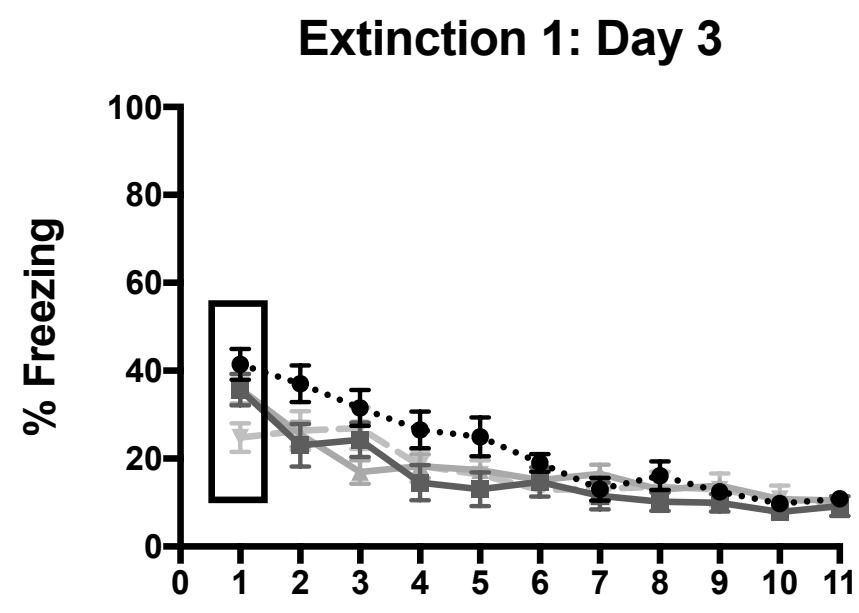

1 Minute Bins 


\section{Fear Extinction (Day 4)}

Similarly to the first extinction test on Day 3, there was a significant main effect of extinction over time (within subjects, repeated measure ANOVA, $F(11,473)=16.208, \mathrm{p}<0.001$ ) and no group effect (between subjects, repeated measures of ANOVA, $F(3,43)=1.655$, $p=0.191$ ). All subjects had significantly extinguished fear by the end of the first extinction trial and showed very low rates of freezing at the start of the second extinction test. See Figure 7.

Figure 7: Depicts all treatment groups extinguishing fear to pre-conditioning levels of fear expression.

Figure 7

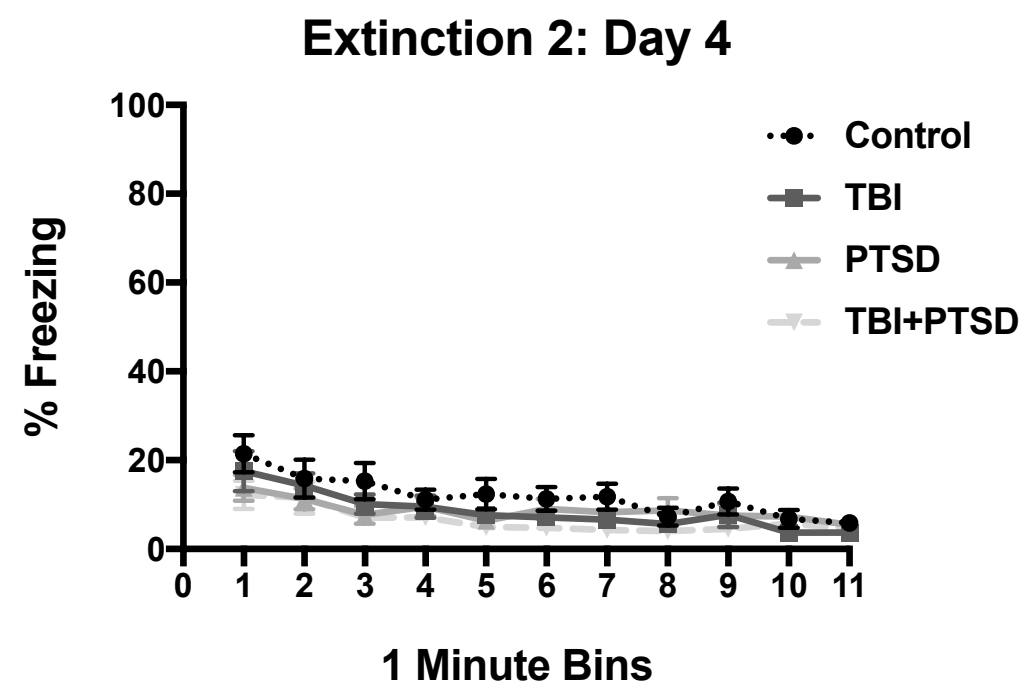




\section{Discussion}

Our data revealed that TBI+PTSD, represented in this study by combining CCI and SPS, produced an inhibited ability to recall previously learned CS and US associations during fear recall tests. PTSD mice over-generalized learned fear to a neutral context more than TBI alone and control groups indicating that this symptom in a combined TBI+PTSD model is likely driven by PTSD. The TBI+PSTD group did not show an increased or decreased sensitivity to fear conditioning or any noticeable difference in ability to extinguish learned fear.

In this study we set out to learn about fear learning and behavior in a combined TBI and PTSD mouse model. There has been a push to learn more about TBI+PTSD because of the high rates of co-occurrence, especially in the veteran population, and the lack of research. Findings from this study are useful for developing a mouse model to further research the pathophysiology of TBI+PTSD and to inform a deeper understanding of the unique behavioral phenotype this model produces. As far as the author has investigated, this study is the first to combine CCI and SPS to create a novel TBI+PTSD mouse model. The sample consisted of 48 mice randomized to one of four groups: control, TBI, TBI+PTSD, and PTSD. After receiving their assigned treatment based on group all mice went through four days of behavioral tests. Fear conditioning was used to examine fear behavior because it involves overlapping neural circuitry involved in TBI and PTSD and is conserved across species.

Results from the behavioral tests revealed that TBI+PTSD group showed significantly decreased ability to recall the conditioning context upon re-exposure. The delayed recollection of the previously learned CS and US association is likely reflective of the neural circuitry implicated in TBI and PTSD: Amygdala, hippocampus, and the PFC, which are also central to emotional memory formation, storage, and recall. Bryant (2011) discusses the impact of TBI on the 
neurological mechanisms responsible for encoding the traumatic memory, often resulting in an inaccurate re-creation of the events leading up to their traumatic incident. Our findings appear to support Bryant (2011) because the mice in this study displayed significantly impaired ability to recall the CS 48 hours after conditioning. Further research is necessary to better understand this deficit in fear recall.

An important study conducted by Palmer, Metheny, Elkind, \& Cohen (2016) provides support for a shared neuroanatomical substrate for TBI and PTSD. They employed lateral fluid percussion injury (LFPI) as a mouse model of mild TBI to examine its effect on amygdala function during behavioral and physiological tests. Their findings suggest that mild TBI causes significant alterations within amygdala circuitry that are correlated with the neuropsychiatric symptoms commonly seen in this diagnosis. Similarly, because the amygdala is responsible for the processing of emotional stimuli that triggers an automatic or conscious response any alterations in this circuitry is likely responsible for the high comorbidity seen with TBI and PTSD (Palmer et al., 2016). The researchers acknowledge that their findings oppose other studies looking at mild TBI that have found increased fear conditioning and amygdala excitability. The Palmer et al (2016) injured mice exhibited decreased rates of freezing during cued fear conditioning tests and decreased amygdala excitation during physiological tests. The researchers suggest their findings may not be contradictory but represent the different presentation of symptoms seen in TBI patients (Palmer et al., 2016).

Reger et al (2012) set out to examine the link between TBI and the increased risk of developing PTSD in a rat model. This study was specifically mentioned by Palmer et al (2016) as having opposing findings to their own. Their findings suggested that TBI caused neuroanatomical alterations which resulted in significant increases in fear learning as well as, an over-generalized 
learned fear response. Another study, Meyer et al (2012) employed a rat model of mild TBI to investigate their hypothesis that alterations in neuroanatomy caused by TBI can result in PTSD or other anxiety-like behaviors. They found that mild TBI causes an increase in amygdala volume as well as, in unconditioned fear behavior and conditioned fear learning. Furthermore, the researchers did not find an effect on fear extinction, implying that the impairment seen in fear extinction is likely driven by PTSD (Meyer et al., 2012). While studies have found differences in fear behavior and expression in mild TBI subjects they all support that TBI has a significant effect on the amygdala (Palmer et al., 2016; Reger et al., 2012; Meyer et al., 2012).

During fear conditioning and fear extinction all treatment groups were able to acquire and extinguish fear. The combined TBI+PTSD group did not show a heightened sensitivity to conditioning as reported by Meyer et al (2012) and Reger et al (2012), or a decreased sensitivity as observed by Palmer et al (2016). These findings support the claim made by Palmer et al (2016) that differences seen among experimenters are likely the reflection of the different presentations in symptomatology observed in the TBI disorder based on severity. This brings up a question as to which TBI model results in the most representative TBI for a combined TBI+PTSD model. TBI and PTSD did not affect fear extinction, all groups were able to extinguish fear to pre-conditioning levels of fear expression by the end of the second extinction trial. This data supports Meyer et al (2012) who found no effect on fear extinction in a TBI model.

Sierra-Mercado et al (2013) combined CCI as a TBI mouse model with fear conditioning and extinction to examine the relationship between TBI and PTSD. Their findings suggest that CCI does not affect fear conditioning or extinction within two-weeks of injury. The researchers question CCI as a TBI model for studying fear learning and memory because they were unable to 
find a relationship between "contusion TBI", or CCI, and fear learning and extinction. They suggest that a TBI model that produces a concussive type TBI such as Lateral Fluid Percussion Injury may be more appropriate for studying fear learning and memory because this is more in line with the type of TBI typically diagnosed with PTSD (Sierra-Mercado et al, 2013). Similar to Sierra-Mercado et al (2013) our CCI model combined with SPS did not impairments in fear conditioning or extinction tests. Together these findings lend further support for the need to investigate which TBI model is best for a combined TBI+PTSD model.

Xing et al (2013) examined biological mechanisms that produce TBI+PTSD pathologies using Fluid Percussion Injury as a TBI model and repeated tail shocks as a PTSD model. Their findings suggest that exposure to repeated stress or mild TBI alone increases anxiety and impairs memory and that this effect was long-lasting. Contrary to their findings, our TBI+PTSD group did not express increased anxiety or sensitivity to fear conditioning, increased fear expression in the context generalization test, or difficulty extinguishing fear. However, we did observe impaired memory in the TBI+PTSD group but, unlike Xing et al (2103) we only observed impaired fear recall in the combined group.

Acosta et al (2013) combined CCI with a different PTSD model then the one used in this study to investigate the histopathological connection between TBI and PTSD. Their findings suggest that PTSD does not influence neuroinflammation or neurodegeneration triggered by TBI. The neurodegeneration seen within the hippocampus in the combined TBI+PTSD model (Acosta et al., 2013) may account for a weak formation of the CS-US association because the hippocampus is responsible for initially encoding these associations. A weak formation of the CS-US association may produce delayed recall of the conditioning chamber similar to what was observed in our study during the fear recall test. These findings lend support to researching the 
effects of a stronger CS-US association in a combined TBI+PTSD group on fear learning and extinction.

Due to using a mouse model for our study findings may not translate to humans and other rodent models. All experiments and observations were performed in the controlled environment of a laboratory. As a controlled study there are limitations on generalizing results outside of a controlled laboratory. The severity of TBI delivered by the CCI model used in this study may be responsible for producing the observed impairment in fear recall from the TBI+PTSD group. We intend on looking at brain histology to determine potential correlates between injury size/location and behavior. If the severity of the CCI injury is responsible for the deficit then it would imply results are only generalizable to TBIs of similar severity. Another factor the experimenters would like to investigate is if the context generalization test may have acted as a brief extinction trial due to the subtle similarities between the conditioning context and the altered context used for the test. If the context generalization test acted as an initial fear extinction trial it might explain why there were low rates of freezing during the fear recall tests and is worth researching further. Finally, while all groups significantly acquired fear during fear conditioning tests rates of freezing never reached what would be considered very high (one group, PTSD, reached a high of 50-60\% freezing per minute by ITI 5). It may be worth examining and making adjustments to the conditioning trial to encourage subjects form a stronger CS and US association.

In culmination these data provide useful information for the development of a novel mouse model of TBI+PTSD to further research this commonly co-occurring diagnosis. A mouse model is a valuable asset for learning the pathophysiology of disorders such as TBI and PTSD and has an important role in informing better treatments. Findings from this study suggest that exposure therapy which is the standard treatment for PTSD should be just as effective at extinguishing fear 
with a comorbid diagnosis of TBI and PTSD. However, there is evidence that TBI may result in reconstruction of inaccurate representations of the traumatic experience, which should be investigated. Inaccurate reconstructions that are responsible for fear memories should be used for fear extinction/exposure therapy regardless of their accuracy. Previous research has shown that exposure therapy was successful at treating individuals with TBI+PTSD and inaccurate reconstructed memories of the trauma (Bryant, 2011). This study provides support for the importance of considering TBI severity in regard to symptom presentation and treatment.

\section{Conclusion}

Data from this research indicates that TBI interacts with PTSD to impair fear recall of conditioned associations that have an initial impact on fear extinction. However, there was not an effect on fear extinction over time as all groups, TBI+PTSD included, were able to extinguish fear to pre-fear conditioned levels of fear expression. This result may be due to the mild nature of our model. Further modifications need to be researched to examine a potential dose response of injury on fear behavior and learning. Our findings lend support to exposure therapy as an effective treatment for a comorbid TBI and PTSD diagnosis. TBI+PTSD treatments did not interact to have an effect on fear conditioning, all four groups increased rates of fear expression across ITIs at similar rates. The study presented here provides data on a novel mouse model of TBI+PTSD using CCI and SPS to represent each disorder, respectively, which successfully produced behaviors that are commonly associated with each. Therefore, this study generated a useful experimental model of TBI+PTSD by combining CCI and SPS that can be used in a laboratory setting to further research this common and unique bio-psycho-social phenotype. 


\section{References}

Acosta, S., Diamond, D.M., Wolfe, S., Tajiri, N., Shinozuka, K., Ishikawa, H...Borlorgan, C.V. (2013). Influence of Post-Traumatic Stress Disorder on Neuroinflammation and Cell Proliferation in a Rat Model of Traumatic Brain Injury. PLoS One, 8(12) doi:http://dx.doi.org.proxy.lib.pdx.edu/10.1371/journal.pone.0081585

Amano, T., Unal, C. T., \& Paré, D. (2010). Synaptic correlates of fear extinction in the amygdala. Nature Neuroscience, 13(4), 489-494. https://doi.org/10.1038/nn.2499

An, B., Hong, I., \& Choi, S. (2012). Long-Term Neural Correlates of Reversible Fear Learning in the Lateral Amygdala. Journal of Neuroscience, 32(47), 16845-16856. https://doi.org/10.1523/JNEUROSCI.3017-12.2012

Baker, K. D., \& Richardson, R. (2015). Forming competing fear learning and extinction memories in adolescence makes fear difficult to inhibit. Learning \& Memory, 22(11), 537-543. https://doi.org/10.1101/lm.039487.114

Blanchard, R.J. \& Blanchard, C. D. (1969). Crouching As An Index Of Fear. Journal of Comparative and Physiological Psychology, (7)3, 370-375.

Bouton, M.E. (2002). Context, Ambiguity, and Unlearning: sources of relapse after extinction. Biological Psychiatry, 52:976-986. Retrieved November 3, 2017, from http://www.sciencedirect.com.proxy.lib.pdx.edu/science/article/pii/S0006322302015469

Bouton, M.E., Westbrook, R.F., Corcoran, K. A., Maren, S. (2006). Contextual and Temporal Modualtion of Extinction: Behavioral and Biological Mechanisms. Biological Psychiatry, 60, 352-360. DOI:10.1016/j.biopsych.2005.12.015 
Debiec, J., Diaz-Mataix, L., Bush, D. E. A., Doyère, V., \& LeDoux, J. E. (2013). The selectivity of aversive memory reconsolidation and extinction processes depends on the initial encoding of the Pavlovian association. Learning \& Memory, 20(12), 695-699. https://doi.org/10.1101/1m.031609.113

Feder, A., Nestler, E. J., \& Charney, D. S. (2009). Psychobiology and molecular genetics of resilience. Nature Reviews Neuroscience, 10(6), 446-457. https://doi.org/10.1038/nrn2649

Gao, J., Wang, H., Liu, Y., Li, Y., Chen, C., Liu, L., ... Yang, C. (2014). Glutamate and GABA imbalance promotes neuronal apoptosis in hippocampus after stress. Medical Science Monitor: International Medical Journal of Experimental and Clinical Research, 20, 499512. https://doi.org/10.12659/MSM.890589

Katz, D.I., Cohen, S.I., \& Alexander, M.P. (2015). Chapter 9: Mild traumatic brain injury. Handbook of Clinical Neurology, 127 (3 ${ }^{\text {rd }}$ series), 131-156. DOI:10.1016/B978-0-444$52892-6.00009-X$

Lim, S., Song, K., Yoo, C., Woo, D., \& Choe, B. (2017). Decreased glutamatergic activity in the frontal cortex of single prolonged stress model: in vivo and ex vivo proton MR spectroscopy. Neurochemical Research, 42 (8) 2218-229 DOI:10.1007/s11064-017-2232-x

Mahan, A.L. \& Ressler, K.J. (2012). Fear conditioning, synaptic plasticity, and the amygdala: implications for posttraumatic stress disorder. Trends in Neuroscience, 35(1), 24-35. https://doi.org/10.1016/j.tins.2011.06.007

Maren, S. (2001). Neurobiology of Pavlovian Fear Conditioning. Annual Review of Neuroscience, 24(1), 897-931. https://doi.org/10.1146/annurev.neuro.24.1.897 
Maren, S., Phan, K. L., \& Liberzon, I. (2013). The contextual brain: implications for fear conditioning, extinction and psychopathology. Nature Reviews Neuroscience, 14(6), 417428. https://doi.org/10.1038/nrn3492

The article, 'The contextual brain', reviews current research related to fear conditioning and extinction in the field of behavioral neuroscience. The first author, Stephen Maren, has many publications in this area and has contributed significantly to this field's understanding of Pavlovian Fear Conditioning. The goal of this work is to inform treatment and health policies for anxiety disorders such as Post-Traumatic Stress disorder and Generalized Anxiety Disorder. Specifically, in this article the authors aim to summarize relevant research related to the terms commonly used in this field, the models used to test fear learning/extinction, and important findings from research already completed. The article is from Nature Review: Neuroscience and is a secondary source because it is a meta-analysis of research already conducted. Information important in this article relevant to the specific research question of this current study included: operational definitions for common terms used in this area of study and explanations of the anatomical structures involved. Also, this article describes experimental models commonly used in fear learning and extinction tests.

Maren, S., \& Quirk, G. J. (2004). Neuronal signaling of fear memory. Nature Reviews Neuroscience, 5(11), 844-852. https://doi.org/10.1038/nrn1535

McAllister, T.W. (2009). Psycholopharmacological Issues in the treatment of TBI and PTSD. The Clinical Neuropsychologist, 23(8), 1338-1367. https://doi.org/10.1080/13854040903277289

Meyer, D. L., Davies, D. R., Barr, J. L., Manzerra, P., \& Forster, G. L. (2012). Mild traumatic brain injury in the rat alters neuronal number in the limbic system and increases conditioned fear and anxiety-like behaviors. Experimental Neurology, 235(2), 574-587. https://doi.org/10.1016/j.expneurol.2012.03.012

This study is a collaboration between researchers at Sanford School of Medicine, University of South Dakota and Department of Pharmacology, Temple University School of Medicine, Philadelphia. Support came from the National Institute of Health, Department of Defense, and miscellaneous affiliated institutional research grants. This 
article has been peer-reviewed and published in the journal, Experimental Neurology, in 2012. The researchers hypothesized that $m$ TBI results in changes to the limbic system which is responsible for the increased fear and anxiety commonly seen in mTBI. To test hypothesis a rat model of mTBI was employed that the researchers called a "modified weight drop method" which they felt more closely mimicked a mTBI or concussion. They also performed multiple behavioral tests then followed with looking at brain histology. The behavioral tests performed included contextual fear conditioning, locomotor, elevated plus maze, rotarod, and tail-withdrawal tests. The conditioning was performed similarly to our own study, 8 days following mTBI subjects were placed in conditioning chamber where US was delivered (a foot-shock of $0.75 \mathrm{~mA}$, for 2 second duration, with 75 seconds between deliveries for a total of 10 shocks delivered). They found that mTBI subjects had increased sensitivity to fear conditioning as well as to unconditioned fear, however there was no effect observed on fear extinction. Brain histology revealed that the hippocampus endures cell loss after injury however, for the first time they showed that the amygdala has an increase in cell number after injury. A decrease in cell apoptosis and an increase in cell production enlarges the volume of the amygdala. These findings support the findings from a previous study that found enlarged amygdala volume among children diagnosed with TBI 10 years post injury. Interestingly, the researchers pointed out that increases in amygdala volume may be seen as a result of both positive and negative experiences, citing a study that found these results in mice who were exposed to enriched environments. Together, these findings support the researchers' hypothesis that changes within the limbic system produce increases in fear and anxiety-like behaviors after mTBI.

Orsini, C.A., Kim, J.H., Knapske, E., \& Maren, S. (2011). Hippocampal and Prefrontal Projections to the Basal Amygdala Mediate Contextual Regulation of Fear after Extinction. The Journal of Neuroscience, 31(47), 17269-17277.

DOI:10.1523/JNEUROSCI.4095-11.2011

Orsini, C.A., and Maren, S. (2012). Neural and Cellular Mechanisms of Fear and Extinction Memory Formation. Neuroscience and Behavioral Reviews, 36, 1773-1802.

DOI:10.1016/j.neubiorev.2011.12.014

Palmer, C.P., Metheny, H.E., Elkind, J.A, \& Cohen, A.S. (2016). Diminished amygdala activation and behavioral threat response following traumatic brain injury. Experimental Neurology, 277, 215-226. http://dx.doi.org/10.1016/j.expneurol.2016.01.004 
Perez-Garcia, G., Gama Sosa, M. A., De Gasperi, R., Lashof-Sullivan, M., Maudlin-Jeronimo, E., Stone, J. R., ... Elder, G. A. (2016). Chronic post-traumatic stress disorder-related traits in a rat model of low-level blast exposure. Behavioural Brain Research. https://doi.org/10.1016/j.bbr.2016.09.061

Pibiri, F., Nelson, M., Guidotti, A., Costa, E., \& Pinna, G. (2008). Decreased corticolimbic allopregnanolone expression during social isolation enhances contextual fear: A model relevant for posttraumatic stress disorder. Proceedings of the National Academy of Sciences, 105(14), 5567-5572. https://doi.org/10.1073/pnas.0801853105

Reger, M. L., Poulos, A. M., Buen, F., Giza, C. C., Hovda, D. A., \& Fanselow, M. S. (2012). Concussive Brain Injury Enhances Fear Learning and Excitatory Processes in the Amygdala. Biological Psychiatry, 71(4), 335-343.

https://doi.org/10.1016/j.biopsych.2011.11.007

This study was conducted in 2012 at the Neurotrauma lab, University of California at Los Angeles, and the researchers themselves are neurologists, neuropsychologists, and behavioral neuroscientists. The research is supported by the Department of Defense, National Institute of Health, and the UCLA Brain Injury Research Center. The authors recognizing a high correlation between TBI and PTSD set out to examine the link. Because of the high emotional intensity typically involved in circumstances leading TBI it is difficult to decipher if TBI alone results in increased fearlanxiety or if this is driven by the psychological stress. The researchers hypothesized that TBI results in neuroanatomical changes that effect excitatory and inhibitory processes therefore, increasing risk of neuropsychopathology. To test their hypothesis, they utilized a rat model of TBI, lateral fluid percussion injury (LFPI) and Pavlovian Fear Conditioning as well as, performed brain histology. Their findings supported their hypothesis, subjects exhibited increased rates of conditioned fear and increased rates of fear to novel contexts (fear generalizing). Also, brain histology revealed changes within the amygdala (increased plasticity and excitation) suggestive of long-term alterations in neural circuitry after mTBI. 
Richardson, L.K., Frueh, B.C., \& Acierno, R. (2010). Prevalence estimates of combat-related post-traumatic stress disorder: critical review. Australian and New Zealand Journal of Psychiatry, 44(1), 4-19. DOI:10.3109/00048670903393597

Schafe, G. E., Nader, K., Blair, H. T., \& LeDoux, J. E. (2001). Memory consolidation of Pavlovian fear conditioning: a cellular and molecular perspective. Trends in Neurosciences, 24(9), 540-546. https://doi.org/10.1016/S0166-2236(00)01969-X

Siegmund, A., \& Wotjak, C. T. (2007). A mouse model of posttraumatic stress disorder that distinguishes between conditioned and sensitised fear. Journal of Psychiatric Research, 41(10), 848-860. https://doi.org/10.1016/j.jpsychires.2006.07.017

US Department of Veterans Affairs. (2016). PTSD: National Center for PTSD. Retrieved from https://www.ptsd.va.gov/public/ptsd-overview/basics/how-common-is-ptsd.asp

Sierra-Mercado, D., McAllister, L.M., Lee, C.H.C., Milad, M.R., Eskandar, E.N., \& Whalen, M.J. (2015). Controlled cortical impact before or after fear conditioning does not affect fear extinction in mice. Science Direct, Vol 1606, 133-141 http://dx.doi.org/10.1016/j.brainres.2015.02.031 0006-8993/

Xiong, Y., Mahmood, A., \& Chopp, M. (2013). Animal models of traumatic brain injury. Nature Reviews: Neuroscience 14, 128-142. DOI.10.1038/nrn3407

Xing, G., Barry, E.S., Benford, B., Grunberg, N.E., Li, H., Watson, W.D., \& Sharma, P. (2013). Impact of repeated stress on traumatic brain injury-induced mitochondrial electron transport chain expression and behavioral responses in rats. Frontiers in Neurology: Neurotrauma Vol 4(196), 1-13 
Yamamoto, S., Morinobu, S., Takei, S., Fuchikami, M., Matsuki, A., Yamawaki, S., \& Liberzon, I. (2009). Single prolonged stress: toward an animal of posttraumatic stress disorder. Depression and Anxiety: review 26, 1110-1117. DOI.10.1002/da.20629

Yehuda, R. (2002). Post-Traumatic Stress Disorder. The New England Journal of Medicine, 346(2), 108-114. https://doi.org/10.1056/NEJMra012941 